\title{
Importance of the hexagonal lipid phase in biological membrane organization
}

\author{
Juliette Jouhet ${ }^{1,2,3,4}$ * \\ ' Laboratoire de Physiologie Cellulaire et Végétale, UMR 5168, CNRS, Grenoble, France \\ ${ }^{2}$ Laboratoire de Physiologie Cellulaire et Végétale, Univ. Grenoble Alpes, Grenoble, France \\ ${ }^{3}$ Laboratoire de Physiologie Cellulaire et Végétale, Institut de Recherches en Technologies et Sciences pour le Vivant, Direction des Sciences du Vivant, \\ Commissariat à l'Energie Atomique et aux Energies Alternatives, Grenoble, France \\ ${ }^{4}$ Laboratoire de Physiologie Cellulaire et Végétale, USC1359, Institut National de la Recherche Agronomique, Grenoble, France
}

\section{Edited by:}

Emmanuelle Bayer, Centre National de la Recherche Scientifique, France

\section{Reviewed by:}

Gian Pietro Di Sansebastiano, Università del Salento, Italy

Laure Béven, University of Bordeaux INRA, UMR 1332 BFP, France

\section{*Correspondence:}

Juliette Jouhet, Laboratoire de Physiologie Cellulaire et Végétale (LPCV), CNRS (UMR5168) / Univ. Grenoble Alpes / INRA (USC1359) / CEA Grenoble, Institut de Recherches en Technologies et Sciences pour le Vivant (iRTSV), 17 rue des Martyrs, 38054 Grenoble Cedex 9, France e-mail: juliette.jouhet@cea.fr
Domains are present in every natural membrane. They are characterized by a distinctive protein and/or lipid composition. Their size is highly variable from the nano- to the micrometer scale. The domains confer specific properties to the membrane leading to original structure and function. The determinants leading to domain organization are therefore important but remain obscure. This review presents how the ability of lipids to organize into hexagonal II or lamellar phases can promote particular local structures within membranes. Since biological membranes are composed of a mixture of lipids, each with distinctive biophysical properties, lateral and transversal sorting of lipids can promote creation of domains inside the membrane through local modulation of the lipid phase. Lipid biophysical properties have been characterized for long based on in vitro analyses using non-natural lipid molecules; their re-examinations using natural lipids might open interesting perspectives on membrane architecture occurring in vivo in various cellular and physiological contexts.

\section{Keywords: glycerolipid, lipid bilayers, hexagonal phase, membrane domains, lipid phase}

Domains are present in every natural membrane. They are characterized by a distinctive protein and/or lipid composition and they confer specific properties to the membrane leading to original structure and function. Plasmodesmata, a highly specialized membrane organization that connects two plant cells, involves membrane domains. This typical plant structure is composed of two membranes: the plasma membrane and the desmotubule, a narrow tube in continuity with the endoplasmic reticulum (ER). Membrane domains described as lipid raft were found in the plasma membrane of plasmodesmata and might be involved in plasmodesmata scaffolding but nothing is known about the lipid organization of the desmotubule. The diameter of the membrane desmotubule is between 10 and $15 \mathrm{~nm}$, which is highly constricted for a bilayer (Tilsner etal., 2011). Continuity of the membrane and of the luminal space between reticulum and desmotubule is now clearly established (Tilsner et al., 2011) but the organization of the desmotubule membrane as a bilayer has never been demonstrated. The presence of non-bilayer phase in desmotubule as a hypothesis may provide a new angle for desmotubule model establishment. This review presents how the ability of lipids to organize into non-lamellar phases, particularly hexagonal II (HII) phase, can promote specific local structures within membranes.

Works on lipid membrane organization were first done by physical chemistry using synthetic lipids and model membranes but they gave us the premises for apprehending the biology of cell membrane structure. According to the Singer and Nicholson's model (Singer and Nicolson, 1972), cell membranes are viewed as proteins embedded in a lipid matrix. This so-called mosaic fluid model includes two basic postulates referring to the "lipid phase" state - liquid crystalline and bilayer, both of which are of vital importance for the proper functioning of membranes. In in vitro systems, aqueous dispersions of lipids are however able to form a large variety of other phases such as non-liquid crystalline and non-bilayer phases. These "solid phases" (also called "gel phases") are favored by low temperature and long and saturated fatty acid chains. They were mainly characterized on model membranes of saturated phosphatidylcholine (PC; for example, see Mason, 1998). However, natural lipids are mostly unsaturated and organisms adapt their fatty acid composition to the environment to prevent the formation of gel phases. Moreover, even though gel phase domains were detected in biological membranes in very specific cases such as the myelin sheath (Ruocco and Shipley, 1984) or in the stratum corneum (Norlen, 2001b), most biological membranes are organized in liquid phase. Therefore gel phases will not be described further in this review.

Since the mosaic fluid model largely neglected the possibility that lipids are not randomly distributed in the bilayer and also understated the degree of local order that can be generated in membranes, this model was soon enriched with the introduction of the membrane domain concept (for a review on membrane model history, see Edidin, 2003). The domains, identified at first in vitro in model membranes (Jain and White, 1977), were further confirmed in vivo with the raft concept (Simons and Ikonen, 1997) and defined as patches of lipids with composition and physical state that differed from the average. 
The raft domains that involve also sterols and sphingolipids and correspond to patches of lipids, in a liquid ordered phase, within a matrix in a liquid disordered phase, have been referenced in depth in different reviews (Bagatolli et al., 2010; Simons and Sampaio, 2011) and will not be described further here. This review will consider more specifically domains resulting from modification of glycerolipid biophysical organization since glycerolipids represent the main constituent of the membrane lipid matrix. We shall focus here on the non-bilayer organization that can adopt glycerolipids, their biophysical properties, and their impact on membrane biology, since this topic is rarely raised in recent biological literature.

Membrane glycerolipids are a category of amphiphilic molecules having a 3-carbon glycerol scaffold (each carbon is numbered following the stereospecific numbering nomenclature $s n-1, s n-2, s n-3)$, harboring one or two hydrophobic acyl chains esterified at positions $s n-1$ and $s n-2$, and a hydrophilic polar head at position $s n-3$. Glycerolipids can be separated into two classes in function of their polar head: phospholipids that contain a phosphorous atom and non-phosphorous glycolipids. Major membrane phospholipids found in prokaryotes and eukaryotes are $\mathrm{PC}$, phosphatidylethanolamine (PE), phosphatidylglycerol (PG), diphosphatidylglycerol (DPG) also called cardiolipin, phosphatidylinositol (PI), phosphatidylserine (PS), and phosphatidic acid (PA). Major glycolipids are monogalactosyldiacylglycerol (MGDG), monoglucosyldiacylglycerol (MGlcDG), digalactosyldiacylglycerol (DGDG), diglucosyldiacylglycerol (DGlcDG), and sulfoquinovosediacylglycerol (SQDG). Physical studies showed that the aqueous dispersions of glycerolipid do not always spontaneously form lipid bilayers as it was guessed at first (Gorter and Grendel, 1925). Indeed, the size of the polar head by comparison of the hydrophobic acyl-glycerol backbone affects lipid behavior in aqueous dispersions (Figure 1). By convention, large negative curvature lipids such as MGDG, MGlcDG, PE, DPG, PS, and PA tend to form HII phase or cubic phase, large positive curvature lipids such as lysolipids form hexagonal I (HI) phase whereas small curvature lipids such as DGDG, DGlcDG, SQDG, PC, PG, and PI form lamellar phase, corresponding to the classical bilayer (Shipley et al., 1973; Seddon, 1990; Hansbro et al., 1992; Vikstrom et al., 1999). Bilayers create a planar structure whereas HI phase forms micellar tubules with the polar head on the outside of the tubules and HII phase forms inverted tubules, with the fatty acyl chains pointing toward the outside of tubules and the polar head groups toward the center establishing an aqueous channel (Figure 1).

However, within a class of lipids, fatty acids can also influence the lipid architecture; the effect of increasing chain length and of unsaturation number is expected to favor in general the
MGDG, MGIcDG, PE, PS, PA, DPG

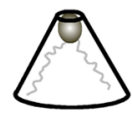

DGDG, DGlcDG, SQDG, PC, PG, PI
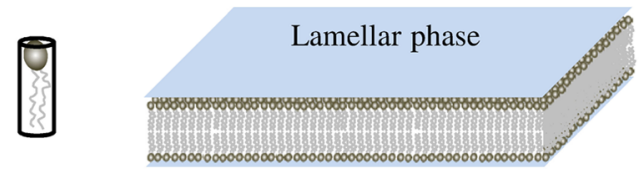

Lysolipid

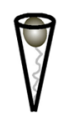

Cubic phase
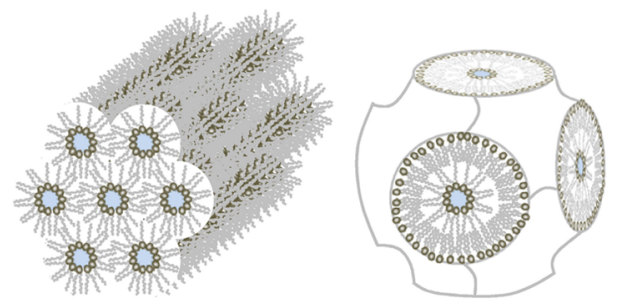

HI phase
FIGURE 1 | Shape structure concept of lipid polymorphism. Lipids with a small polar head have a molecular shape that resembles a truncated cone. They induce a negative curvature strain and favor the organization of membranes into inverted micelles (HII phases) or cubic (bicontinuous) structures. Lipids with a bulky polar head and only one acyl chain have a molecular shape similar to an inverted cone and induce a positive curvature strain in membranes. They favor the formation of tubular (HI) or spheric micelles. Lipids that have similar cross-sectional areas for the polar head and hydrophobic region look like cylinders. They form lamellar phases, with no curvature strain. 
formation of HII phase. For instance, saturated PE form a lamellar phase whereas unsaturated PE form an HII phase (for a review, see Seddon, 1990). Furthermore, HII forming lipids are able to switch from a HII phase to a lamellar phase sometimes through an intermediate cubic phase (Figure 1) by lowering the temperature (Tenchov and Koynova, 2012). Proteins and pigments might also be involved in cubic phase formation (Wang and Quinn, 1999; Almsherqi et al., 2006; Tenchov et al., 2013). All these phase transitions are spontaneous and reversible (Siegel and Tenchov, 2008).

Biological membranes of course contain complex mixtures of lipids, and so it is of great importance to understand the polymorphic phase behavior of such mixtures in well-defined model systems. The use of synthetic lipid mixture and the development of techniques, such as electron microscopy, nuclear magnetic resonance (NMR), X-ray, and neutron diffraction, helped a lot to characterize the parameters that trigger the transition from lamellar phase toward HII phase. Lipid membrane composition, hydration, $\mathrm{pH}$, and presence of cations contribute to lipid organization. For example, an equimolar mixture of $\mathrm{PE}$ and $\mathrm{PC}$ at low hydration pressure is organized in HII phase whereas at high hydration pressure it adopts a bilayer conformation (Ding et al., 2005). Lowering the $\mathrm{pH}$ induces lamellar toward HII transition phase in charged phospholipid system such as PS and PA (Seddon, 1990). Furthermore, transition of DPG from lamellar phase to HII phase is induced either upon lowering $\mathrm{pH}$ to below 2.8, or upon increasing $\mathrm{NaCl}$ concentration to above $1.6 \mathrm{M}$ at $\mathrm{pH} 7$ (Seddon et al., 1983).

The question now is: Do these structures occur in vivo? Most natural membranes are composed of two main glycerolipids: a bilayer forming lipid and a HII forming lipid, respectively the couple PC/PE in yeast and animal cells, PG/PE in Escherichia coli and Bacillus subtilis, DGlcDG/MGlcDG in Acholeplasma laidlawii, and DGDG/MGDG in plants. At a microscopic level, HII structure have been observed in the ER of the retinal pigment epithelium (Yorke and Dickson, 1985) and of the plasma membrane of bladder epithelium (Hicks and Ketterer, 1970). Cubic structures (Figure 1) that are dependent of non-bilayer forming lipids (Siegel, 1999) are also detected in the ER of epidermal keratinocytes (Norlen, 2001a) and in prolamellar bodies in etioplasts (Williams et al., 1998; Gunning, 2001). Furthermore, highly curved membranes like tubules of ER network (Griffing, 2010), the inner mitochondrial membrane (Van Venetie and Verkleij, 1982), or thylakoid grana margins (Murphy, 1982) are thought to be favored by an enrichment in HII forming lipid. At a smaller scale, inverted micellar structures (Figure 2) have been proposed to explain structures observed by NMR in some bilayer systems (for a review see Siegel, 1984).

What is the function of these structures? These structures may be of high importance for some enzyme activities. Enzymes like the calcium pump in the sarcoplasmic reticulum (Yeagle, 1989), the CTP: phosphocholine cytidylyltransferase (Attard et al., 2000) or the violaxanthin de-epoxidase in the thylakoids (Latowski et al., 2004) have an activity dependent of HII structures. Membrane anchoring of some proteins like G-proteins and phosphokinases C is enhanced by HII phase (Escriba etal., 1997; Vogler et al., 2004). Membrane fusion and fission events seem also to be dependent on the presence of non-bilayer forming lipids (for a review, see Burger, 2000). It has been shown that lipid bilayers can fuse in the complete absence of proteins even if membrane fusion is regulated in vivo by specialized proteins. Membrane fusion between phospholipid bilayers can be induced by the HII lipids, PA, and PS, in conjunction with $\mathrm{Ca}^{2+}$ (for a review see Papahadjopoulos et al., 1990) or by dehydration, that drives bilayers into very close contact (Yang and Huang, 2003). All actual models (Chakraborty et al., 2012; Hamilton et al., 2012; Colpitts et al., 2013) for the membrane fusion process share at least one intermediate structure called the fusion stalk (Figure 2; Markin et al., 1984). Stalk formation is promoted by an HII forming lipid like PE whereas it is inhibited by an HI forming lipid like lysoPC (Chernomordik and Kozlov, 2008). The stalk structure is also an intermediate in the lamellar/HII phase transition (Figure 2) and was observed for the first time in a mixture of PE/PC upon dehydration (Yang and Huang, 2002). Parameters affecting the lamellar/HII phase transition can probably be considered also as fusion parameters and biophysical studies of this phase transition, such as calculation of the energy needed for the process, is starting to give a lot of insights on membrane fusion mechanism (Kozlovsky et al., 2004; Pan et al., 2006).

For the preservation of cell structure and compartmentalization, the membrane needs to be in a lamellar phase but, for membrane architecture and for some enzyme activities, HII phase domains must be present. It was shown that bacteria cells are able to keep the membrane lipids in a "window" between lamellar phases and HII phases. For example, E. coli or A. laidlawii maintain a balance between HII forming lipids and bilayer forming lipids by adjusting the composition of the polar head group (A. laidlawii) or the acyl chains (E. coli; Lindblom et al., 2002). This lead to the hypothesis that biomembranes homeostatically adjust their intrinsic curvatures to maintain a constant net spontaneous curvature in each leaflet of the bilayer (Gruner, 1985). Activation of the CTP: phosphocholine cytidylyltransferase by HII phase might be a key factor for this kind of adaptability (Attard et al., 2000). On this model, it was postulated that several enzymes involved in lipid biosynthesis could also be regulated by membrane stored curvature elastic energy. Kinetic simulations of the eukaryotic lipid biosynthetic pathway were used to show how this elastic energy was homeostatically maintained through a HII/bilayer ratio control mechanism (Alley et al., 2008; Beard et al., 2008) similarly to what was proposed for A. laidlawii (Vikstrom et al., 2000).

In conclusion, the biophysical properties of lipids have been characterized for long based on in vitro analyses using non-natural lipid molecules; their re-examinations using natural lipids might open interesting perspectives how membrane structure organizations occur in vivo in various cellular and physiological contexts, like in plasmodesmata. This might comfort the theory that cells adjust their membrane lipid composition in response to perturbations in order to maintain bilayer stability, but keeping the bilayer close to a point of instability, where a confined transformation to some non-bilayer structure would tend to occur. The mechanisms "sensing" the physical state of lipids and regulating the lipid biosynthetic pathways accordingly are unknown. 
A

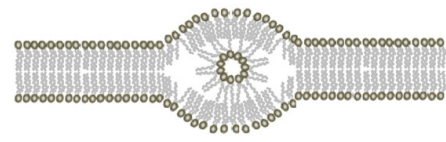

B

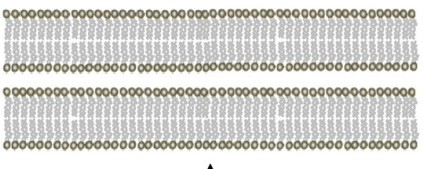

$f$

$2-$

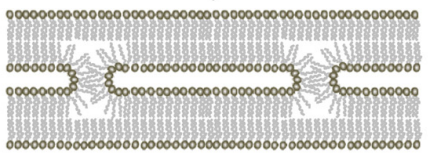

全

$3-$
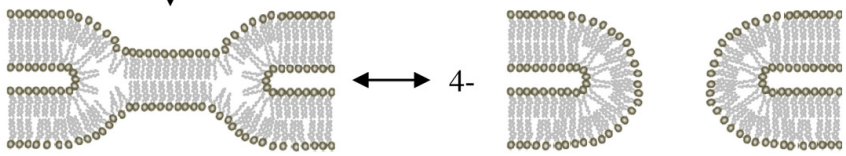

$5-$

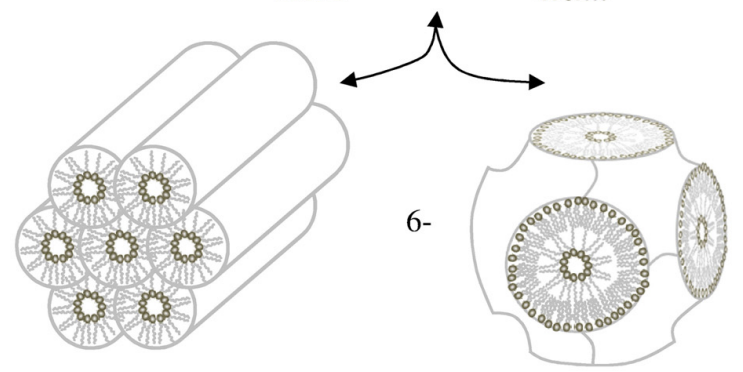

FIGURE 2 | HII phase in bilayers. (A) Lipidic particle as described in (Siegel, 1984). (B) Mechanisms of membrane fusion involving HII via the stalk intermediate. (1) Apposition of two bilayers. (2) Stalk. The stalk is cylindrically symmetrical. (3) Hemifusion intermediate. It can form two different types of structures. If the bilayer diaphragm in the middle of the hemifusion intermediate ruptures, it forms a fusion pore (4) If fusion pores accumulate in sufficient numbers, they can rearrange to form a cubic phase (5) For systems close to the lamellar/HII phase boundary, hemifusion intermediates can also aggregate to form HII phase (6) Figure adapted from Siegel (1999).

\section{ACKNOWLEDGMENTS}

The author was supported by grants from ANR ReGal and ANR ChloroMitoLipid and by the Labex GRAL. The author wishes to thank M. A. Block, B. Demé, and E. Maréchal for fruitful discussions.

\section{REFERENCES}

Alley, S. H., Ces, O., Templer, R. H., and Barahona, M. (2008). Biophysical regulation of lipid biosynthesis in the plasma membrane. Biophys. J. 94, 2938-2954. doi: 10.1529/biophysj.107.118380

Almsherqi, Z. A., Kohlwein, S. D., and Deng, Y. (2006). Cubic membranes: a legend beyond the Flatland ${ }^{*}$ of cell membrane organization. J. Cell Biol. 173, 839-844. doi: $10.1083 / j$ cb. 200603055

Attard, G. S., Templer, R. H., Smith, W. S., Hunt, A. N., and Jackowski, S. (2000). Modulation of CTP: phosphocholine cytidylyltransferase by membrane curvature elastic stress. Proc. Natl. Acad. Sci. U.S.A. 97, 9032-9036. doi: $10.1073 /$ pnas. 160260697

Bagatolli, L. A., Ipsen, J. H., Simonsen, A. C., and Mouritsen, O. G. (2010). An outlook on organization of lipids in membranes: searching for a realistic connection with the organization of biological membranes. Prog. Lipid Res. 49, 378-389. doi: 10.1016/j.plipres.2010.05.001

Beard, J., Attard, G. S., and Cheetham, M. J. (2008). Integrative feedback and robustness in a lipid biosynthetic network. J. R. Soc. Interface 5, 533-543. doi: 10.1098/rsif.2007.1155
Burger, K. N. (2000). Greasing membrane fusion and fission machineries. Traffic 1, 605-613. doi: 10.1034/j.1600-0854.2000.010804.x

Chakraborty, H., Tarafdar, P. K., Bruno, M. J., Sengupta, T., and Lentz, B. R. (2012). Activation thermodynamics of poly(ethylene glycol)-mediated model membrane fusion support mechanistic models of stalk and pore formation. Biophys. J. 102, 2751-2760. doi: 10.1016/j.bpj.2012.04.053

Chernomordik, L. V., and Kozlov, M. M. (2008). Mechanics of membrane fusion. Nat. Struct. Mol. Biol. 15, 675-683. doi: 10.1038/nsmb.1455

Colpitts, C. C., Ustinov, A. V., Epand, R. F., Epand, R. M., Korshun, V. A., and Schang, L. M. (2013). 5-(Perylen-3-yl)ethynyl-arabino-uridine (aUY11), an arabinobased rigid amphipathic fusion inhibitor, targets virion envelope lipids to inhibit fusion of influenza virus, hepatitis C virus, and other enveloped viruses. J. Virol. 87, 3640-3654. doi: 10.1128/JVI.02882-12

Ding, L., Weiss, T. M., Fragneto, G., Liu, W., Yang, L., and Huang, H. W. (2005). Distorted hexagonal phase studied by neutron diffraction: lipid components demixed in a bent monolayer. Langmuir 21, 203-210. doi: 10.1021/la047876u

Edidin, M. (2003). Lipids on the frontier: a century of cell-membrane bilayers. Nat. Rev. Mol. Cell Biol. 4, 414-418. doi: 10.1038/nrm1102

Escriba, P. V., Ozaita, A., Ribas, C., Miralles, A., Fodor, E., Farkas, T., et al. (1997). Role of lipid polymorphism in G protein-membrane interactions: nonlamellar-prone phospholipids and peripheral protein binding to membranes. Proc. Natl. Acad. Sci. U.S.A. 94, 11375-11380. doi: 10.1073/pnas.94.21. 11375

Gorter, E., and Grendel, F. (1925). On bimolecular layers of lipoids on the chromocytes of the blood. J. Exp. Med. 41, 439-443. doi: 10.1084/jem.41.4.439 
Griffing, L. R. (2010). Networking in the endoplasmic reticulum. Biochem. Soc. Trans. 38, 747-753. doi: 10.1042/BST0380747

Gruner, S. M. (1985). Intrinsic curvature hypothesis for biomembrane lipid composition: a role for nonbilayer lipids. Proc. Natl. Acad. Sci. U.S.A. 82, 3665-3669. doi: 10.1073/pnas.82.11.3665

Gunning, B. E. (2001). Membrane geometry of "open" prolamellar bodies. Protoplasma 215, 4-15. doi: 10.1007/BF01280299

Hamilton, B. S., Whittaker, G. R., and Daniel, S. (2012). Influenza virusmediated membrane fusion: determinants of hemagglutinin fusogenic activity and experimental approaches for assessing virus fusion. Viruses 4, 1144-1168. doi: 10.3390/v4071144

Hansbro, P. M., Byard, S. J., Bushby, R. J., Turnbull, P. J., Boden, N., Saunders, M. R., et al. (1992). The conformational behaviour of phosphatidylinositol in model membranes: 2H-NMR studies. Biochim. Biophys. Acta 1112, 187-196. doi: 10.1016/0005-2736(92)90391-X

Hicks, R. M., and Ketterer, B. (1970). Isolation of the plasma membrane of the luminal surface of rat bladder epithelium, and the occurrence of a hexagonal lattice of subunits both in negatively stained whole mounts and in sectioned membranes. J. Cell Biol. 45, 542-553. doi: 10.1083/jcb.45.3.542

Jain, M. K., and White, H. B. III. (1977). Long-range order in biomembranes. $A d v$. Lipid Res. 15, 1-60.

Kozlovsky, Y., Efrat, A., Siegel, D. P., and Kozlov, M. M. (2004). Stalk phase formation: effects of dehydration and saddle splay modulus. Biophys. J. 87, 2508-2521. doi: 10.1529/biophysj.103.038075

Latowski, D., Akerlund, H. E., and Strzalka, K. (2004). Violaxanthin de-epoxidase, the xanthophyll cycle enzyme, requires lipid inverted hexagonal structures for its activity. Biochemistry 43, 4417-4420. doi: 10.1021/bi049652g

Lindblom, G., Oradd, G., Rilfors, L., and Morein, S. (2002). Regulation of lipid composition in Acholeplasma laidlawii and Escherichia coli membranes: NMR studies of lipid lateral diffusion at different growth temperatures. Biochemistry 41, 11512-11515. doi: 10.1021/bi0263098

Markin, V. S., Kozlov, M. M., and Borovjagin, V. L. (1984). On the theory of membrane fusion. The stalk mechanism. Gen. Physiol. Biophys. 3, 361-377.

Mason, J. T. (1998). Investigation of phase transitions in bilayer membranes. Methods Enzymol. 295, 468-494. doi: 10.1016/S0076-6879(98)95054-6

Murphy, D. J. (1982). The importance of non-planar bilayer regions in photosynthetic membranes and their stabilization by galactolipids. FEBS Lett. 150, 19-26. doi: 10.1016/0014-5793(82)81297-0

Norlen, L. (2001a). Skin barrier formation: the membrane folding model. J. Invest. Dermatol. 117, 823-829. doi: 10.1046/j.0022-202x.2001.01445.x

Norlen, L. (2001b). Skin barrier structure and function: the single gel phase model. J. Invest. Dermatol. 117, 830-836.

Pan, D., Wang, W., Liu, W., Yang, L., and Huang, H. W. (2006). Chain packing in the inverted hexagonal phase of phospholipids: a study by X-ray anomalous diffraction on bromine-labeled chains. J. Am. Chem. Soc. 128, 3800-3807. doi: $10.1021 / \mathrm{ja} 058045 \mathrm{t}$

Papahadjopoulos, D., Nir, S., and Duzgunes, N. (1990). Molecular mechanisms of calcium-induced membrane fusion. J. Bioenerg. Biomembr. 22, 157-179. doi: 10.1007/BF00762944

Ruocco, M. J., and Shipley, G. G. (1984). Interaction of cholesterol with galactocerebroside and galactocerebroside-phosphatidylcholine bilayer membranes. Biophys. J. 46, 695-707. doi: 10.1016/S0006-3495(84)84068-0

Seddon, J. M. (1990). Structure of the inverted hexagonal (HII) phase, and nonlamellar phase transitions of lipids. Biochim. Biophys. Acta 1031, 1-69. doi: 10.1016/0304-4157(90)90002-T

Seddon, J. M., Kaye, R. D., and Marsh, D. (1983). Induction of the lamellarinverted hexagonal phase-transition in cardiolipin by protons and mono-valent cations. Biochim. Biophys. Acta 734, 347-352. doi: 10.1016/0005-2736(83) 90134-7

Shipley, G. G., Green, J. P., and Nichols, B. W. (1973). The phase behavior of monogalactosyl, digalactosyl, and sulphoquinovosyl diglycerides. Biochim. Biophys. Acta 311, 531-544. doi: 10.1016/0005-2736(73)90128-4

Siegel, D. P. (1984). Inverted micellar structures in bilayer membranes. Formation rates and half-lives. Biophys. J. 45, 399-420. doi: 10.1016/S0006-3495(84)84164-8

Siegel, D. P. (1999). The modified stalk mechanism of lamellar/inverted phase transitions and its implications for membrane fusion. Biophys. J. 76, 291-313. doi: 10.1016/S0006-3495(99)77197-3
Siegel, D. P., and Tenchov, B. G. (2008). Influence of the lamellar phase unbinding energy on the relative stability of lamellar and inverted cubic phases. Biophys. J. 94, 3987-3995. doi: 10.1529/biophysj.107.118034

Simons, K., and Ikonen, E. (1997). Functional rafts in cell membranes. Nature 387, 569-572. doi: 10.1038/42408

Simons, K., and Sampaio, J. L. (2011). Membrane organization and lipid rafts. Cold Spring Harb. Perspect. Biol. 3, a004697. doi: 10.1101/cshperspect.a004697

Singer, S. J., and Nicolson, G. L. (1972). The fluid mosaic model of the structure of cell membranes. Science 175, 720-731. doi: 10.1126/science.175.4023.720

Tenchov, B., and Koynova, R. (2012). Cubic phases in membrane lipids. Eur. Biophys. J. 41, 841-850. doi: 10.1007/s00249-012-0819-3

Tenchov, B. G., MacDonald, R. C., and Lentz, B. R. (2013). Fusion peptides promote formation of bilayer cubic phases in lipid dispersions. An x-ray diffraction study. Biophys. J. 104, 1029-1037. doi: 10.1016/j.bpj.2012.12.034

Tilsner, J., Amari, K., and Torrance, L. (2011). Plasmodesmata viewed as specialised membrane adhesion sites. Protoplasma 248, 39-60. doi: 10.1007/s00709-0100217-6

Van Venetie, R., and Verkleij, A. J. (1982). Possible role of non-bilayer lipids in the structure of mitochondria. A freeze-fracture electron microscopy study. Biochim. Biophys. Acta 692, 397-405. doi: 10.1016/0005-2736(82)90390-X

Vikstrom, S., Li, L., Karlsson, O. P., and Wieslander, A. (1999). Key role of the diglucosyldiacylglycerol synthase for the nonbilayer-bilayer lipid balance of Acholeplasma laidlawii membranes. Biochemistry 38, 5511-5520. doi: 10.1021/bi982532m

Vikstrom, S., Li, L., and Wieslander, A. (2000). The nonbilayer/bilayer lipid balance in membranes. Regulatory enzyme in Acholeplasma laidlawii is stimulated by metabolic phosphates, activator phospholipids, and double-stranded DNA. J. Biol. Chem. 275, 9296-9302. doi: 10.1074/jbc.275.13.9296

Vogler, O., Casas, J., Capo, D., Nagy, T., Borchert, G., Martorell, G., et al. (2004). The Gbetagamma dimer drives the interaction of heterotrimeric Gi proteins with nonlamellar membrane structures. J. Biol. Chem. 279, 36540-36545. doi: 10.1074/jbc.M402061200

Wang, X., and Quinn, P. J. (1999). Inverted hexagonal and cubic phases induced by alpha-tocopherol in fully hydrated dispersions of dilauroylphosphatidylethanolamine. Biophys. Chem. 80, 93-101. doi: 10.1016/S03014622(99)00063-0

Williams, W. P., Selstam, E., and Brain, T. (1998). X-ray diffraction studies of the structural organisation of prolamellar bodies isolated from Zea mays. FEBS Lett. 422, 252-254. doi: 10.1016/S0014-5793(98)00019-2

Yang, L., and Huang, H. W. (2002). Observation of a membrane fusion intermediate structure. Science 297, 1877-1879. doi: 10.1126/science.1074354

Yang, L., and Huang, H. W. (2003). A rhombohedral phase of lipid containing a membrane fusion intermediate structure. Biophys. J. 84, 1808-1817. doi: 10.1016/S0006-3495(03)74988-1

Yeagle, P. L. (1989). Lipid regulation of cell membrane structure and function. FASEB J. 3, 1833-1842.

Yorke, M. A., and Dickson, D. H. (1985). Lamellar to tubular conformational changes in the endoplasmic reticulum of the retinal pigment epithelium of the newt, Notophthalmus viridescens. Cell Tissue Res. 241, 629-637. doi: 10.1007/BF00214585

Conflict of Interest Statement: The author declares that the research was conducted in the absence of any commercial or financial relationships that could be construed as a potential conflict of interest.

Received: 30 September 2013; accepted: 18 November 2013; published online: 03 December 2013.

Citation: Jouhet J (2013) Importance of the hexagonal lipid phase in biological membrane organization. Front. Plant Sci. 4:494. doi: 10.3389/fpls.2013.00494

This article was submitted to Plant Cell Biology, a section of the journal Frontiers in Plant Science.

Copyright (c) 2013 Jouhet. This is an open-access article distributed under the terms of the Creative Commons Attribution License (CC BY). The use, distribution or reproduction in other forums is permitted, provided the original author(s) or licensor are credited and that the original publication in this journal is cited, in accordance with accepted academic practice. No use, distribution or reproduction is permitted which does not comply with these terms. 